Proceedings

\title{
Research and Development of a Low-Cost Smart Cardio-Pulmonary Resuscitation (CPR) Device Using Locally Available Raw Materials for Cardiac Arrest Patients ${ }^{\dagger}$
}

\author{
Mohammad Monirujjaman Khan * and Md. Mujtabir Alam \\ Department of Electrical and Computer Engineering, North South University, Bashundhara, Dhaka 1229, \\ Bangladesh; mujtabir.alam@northsouth.edu \\ * Correspondence: monirujjaman.khan@northsouth.edu; Tel.: +880-1779006296 \\ + Presented at the 1st International Electronic Conference on Applied Sciences, 10-30 November 2020; \\ Available online: https://asec2020.sciforum.net/.
}

Published: 9 November 2020

\begin{abstract}
Cardiovascular disease is the main worldwide reason for death. Cardiovascular diseases can cause the heartbeat to stop. If a person experiences a cardiac arrest, then direct treatments such as cardio-pulmonary resuscitation (CPR) with chest compressions and artificial ventilation along with defibrillation are methods to greatly improve the patient's possibility of survival. Usually, CPR is completed manually. Manual CPR is carried out by applying external chest compressions followed by artificial ventilation. It helps to pump blood around the person's body when their heart cannot do this job. This paper presents the development and analysis of a low-cost cardio-pulmonary resuscitation (CPR) device using locally available raw materials for the treatment of cardiac arrest patients. This CPR is automated, portable, and very user friendly. This is a very cost-effective product which people can easily afford to buy. The unit price of this CPR is USD 500 .
\end{abstract}

Keywords: cardio-pulmonary resuscitation (CPR); low cost; automated CPR; manual CPR; cardiac patients; multifunction

\section{Introduction}

According to a recent study, the main worldwide reason for death is cardiovascular disease. A total of 17.3 million deaths for each year are identified due to this disease. It is expected that, by 2030, this number may pass more than 23.6 million [1]. In 2008, cardiovascular deaths accounted for 30 percent of every single worldwide demise, with 80 percent of those passing occurring in low- and middle-income nations [2]. The heartbeat of people can be stopped due to cardiovascular diseases. Somebody can stop breathing as well as have heart failure from heart attacks, strokes (when the blood stream to a piece of the brain abruptly stops), choking on something, close suffocating occurrences (when somebody is submerged for a long time and stops breathing), terrible neck, head, or back damage, extreme electrical shocks (such as from touching a power line), being exhausted from a genuine disease, an excessive amount of death, serious unfavorably susceptible responses, and over-consuming a medication or synthetic [3].

Cardio-pulmonary resuscitation (CPR) is a lifesaving medical process that is principally applied to cardiac arrest sufferers. With the end goal of restarting the heart following cardiac arrest, CPR is a well-known technique. When a patient experiences cardiac arrest, then instant action-such as CPR 
with chest compressions and artificial ventilation along with defibrillation-is very likely to greatly improve the patient's probability of survival. At present, conventional Cardio-pulmonary resuscitation (CPR) is manual method and that is applied by medical personnel. It is carried out by applying external chest compressions followed by artificial ventilation. When the heart of the cardiac arrest patient cannot pump the blood around his or her body, CPR assists in pumping blood around the patient's body.

To perform CPR, a person presses up and down on the casualty's chest (chest compressions) and gives them a series of rescue breaths to help save their life when they are in cardiac arrest. The major issue in performing manual CPR is that we are not focusing on teaching the people who most need to be trained to perform this life saving task. It is even very difficult task for trained personnel to maintain constant CPR in a manual process, which significantly reduces the survival chances of the patient [4]. The most generally taught method is something that the huge majority are not able to execute for ten minutes. Many of the people who will be called upon to execute CPR weigh too little to perform 2" chest compressions on a chest of average stiffness. Manual CPR is sometimes not possible to continue perfectly. In this scenario, an automated CPR device can perform constant and proper chest compressions as required for cardiac arrest patients, which may improve their survival rate. In other countries, there are automated CPR devices, but the problem is that they are very expensive and cost between USD 15,000 and USD 20,000 [5,6]. This paper focuses on the development and analysis of a low-cost automated CPR device using locally available raw materials for the treatment of cardiac arrest patients.

\section{Material and Methods}

Figure 1 shows the manual CPR process given by a person. In Figure 2, the automated expensive CPR device available abroad is shown. The objective of this paper is to develop a low-cost automated CPR device using locally available raw materials. Figure 3 shows the block diagram of the working principles of the proposed device. Designing the parts and the working principles of the proposed CPR device are discussed in this section.

This device is automated piston-driven. It provides sternal compressions at the required rate of 100-120 compressions per minute, and completes a compression depth of at least of 2 inches. The operation of the proposed device is easy, as there is no external human being needed to manually perform of the CPR. The proposed device is automated, and is controlled using on and off operation buttons; see the block diagram in Figure 3. First, the device needs to be placed on a stretcher or on the place where the automated CPR will be performed. Then, the proposed device needs to be placed over the chest of the cardiac patient.

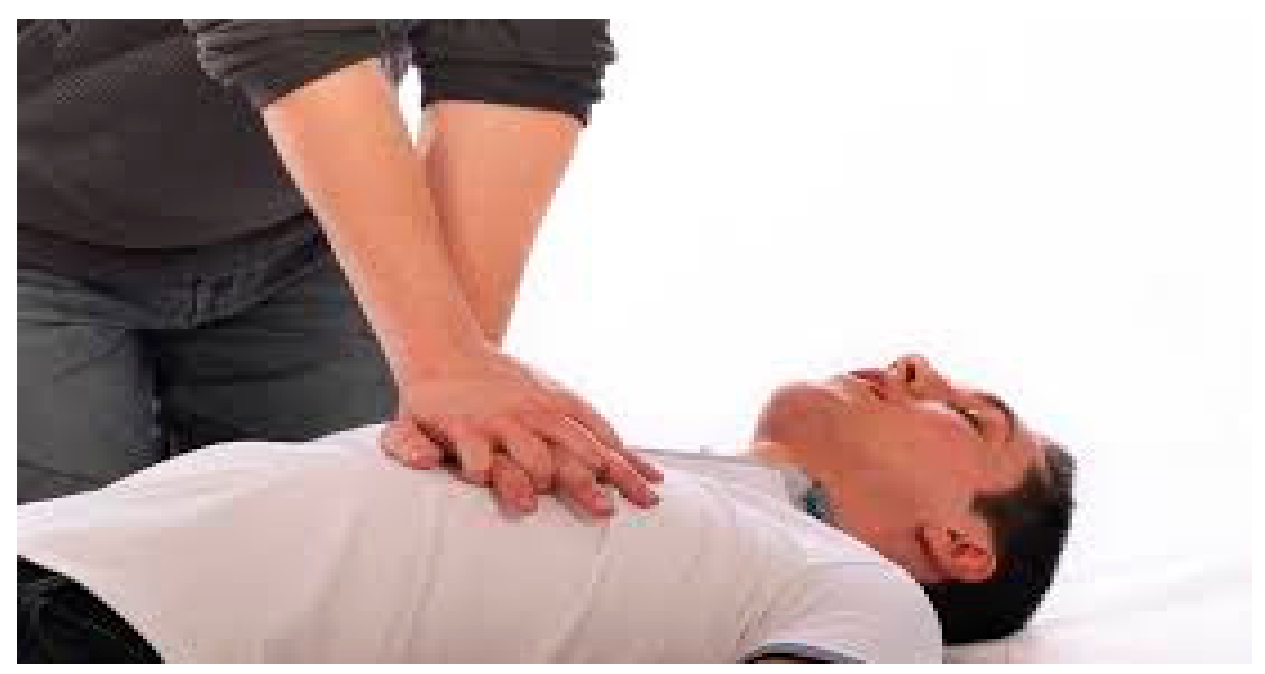

Figure 1. Manual cardio-pulmonary resuscitation (CPR) process given by a person [7]. 


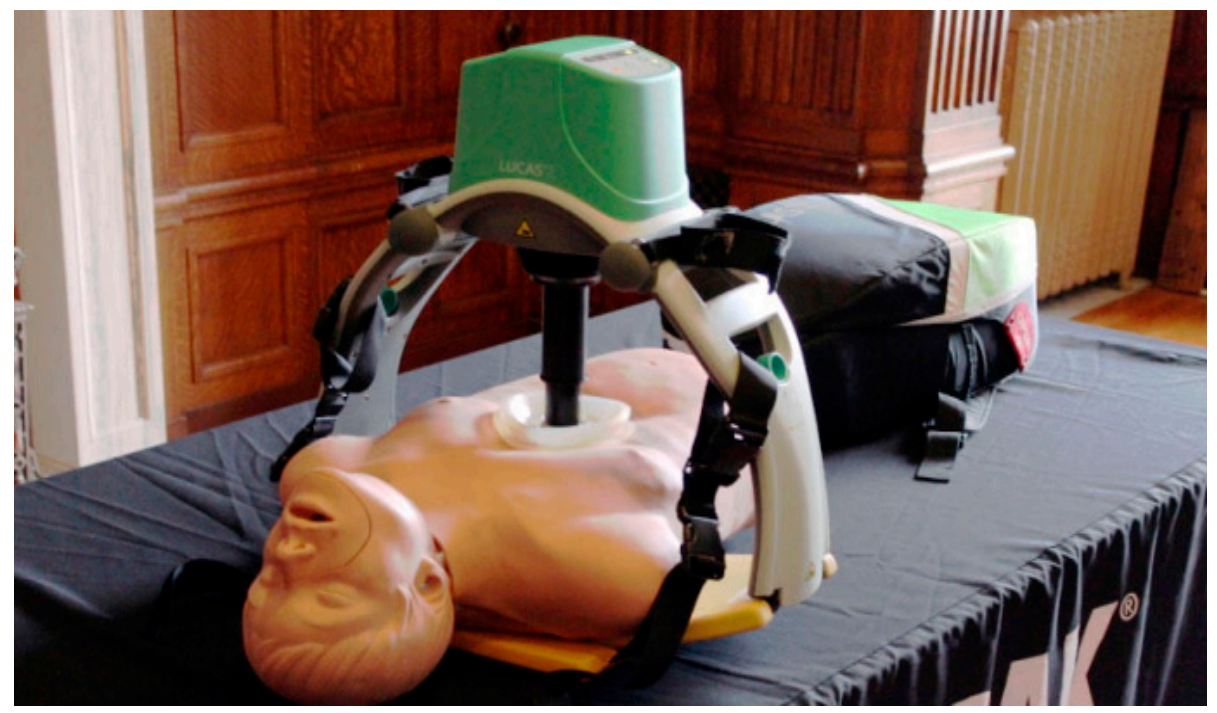

Figure 2. Automated CPR in Abroad [5].

For the operation of the device, three switches have been integrated with the proposed system; see Figure 3. The switches are known as CPR ON/OFF, CPR (30:2), and CPR (Continuous). Different switches have different functions, as can be seen from Figure 3. The on and off switch is connected to an electronic motor controller. This on/off switch is for the powering up and shutting down of the device. When the CPR 30:2 switch is pushed, the device gives 30 chest compressions followed by 2 artificial ventilations. When the CPR (Continuous) switch is pushed, it will provide uninterrupted chest compressions at a rate of 100-120 compressions per minute.

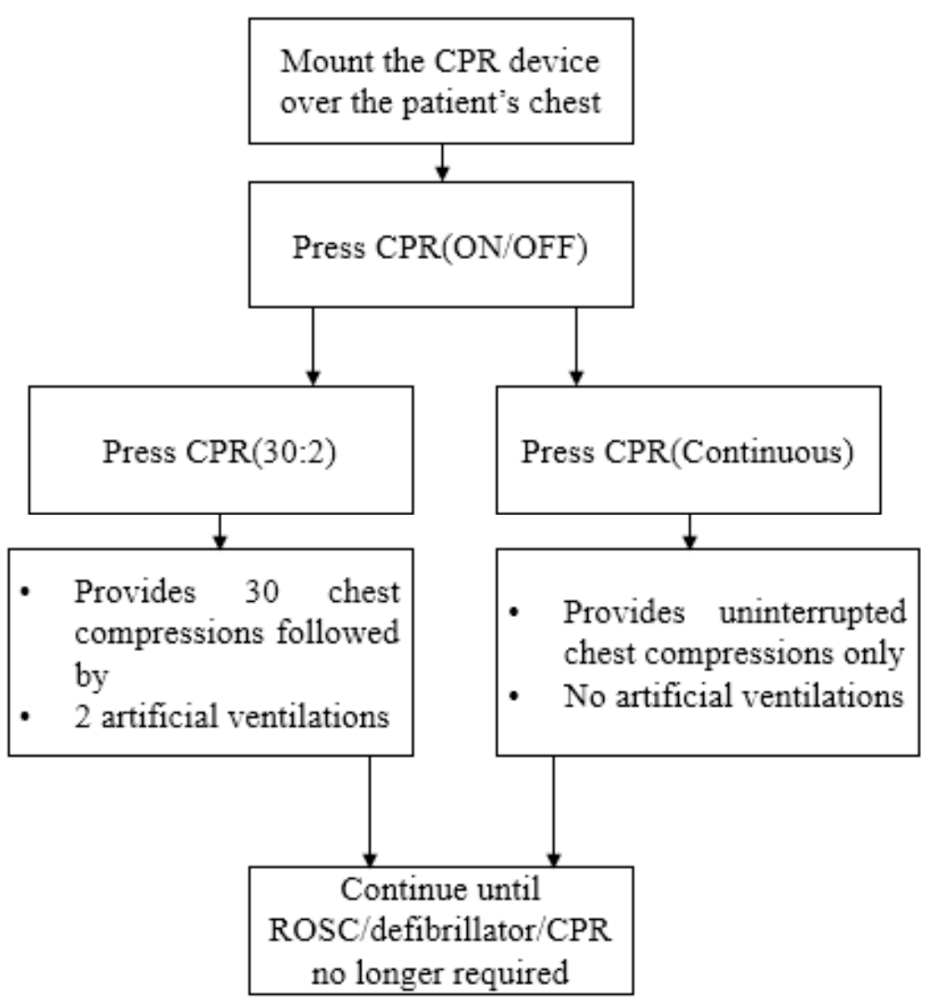

Figure 3. Block diagram of the proposed device how it works. 


\section{Results and Analysis}

Figure 4 demonstrates the prototype of the proposed device. This device was developed in the Engineering Laboratory at the Department of Electrical and Computer Engineering of North South University. The proposed automated device is piston driven-based. It provides sternal compressions at the required rate of 100-120 compressions per minute, and completes a compression depth of at least of 2 inches. The device was made using locally available raw materials procured from the local market. All parts were assembled and tested in the laboratory. The device was designed using a combination of mechanical and electrical components. After the development and integration of the device, the performance of the system was tested on a human-like dummy. Figure 5 provides a finished model of the proposed device showing testing on the dummy.

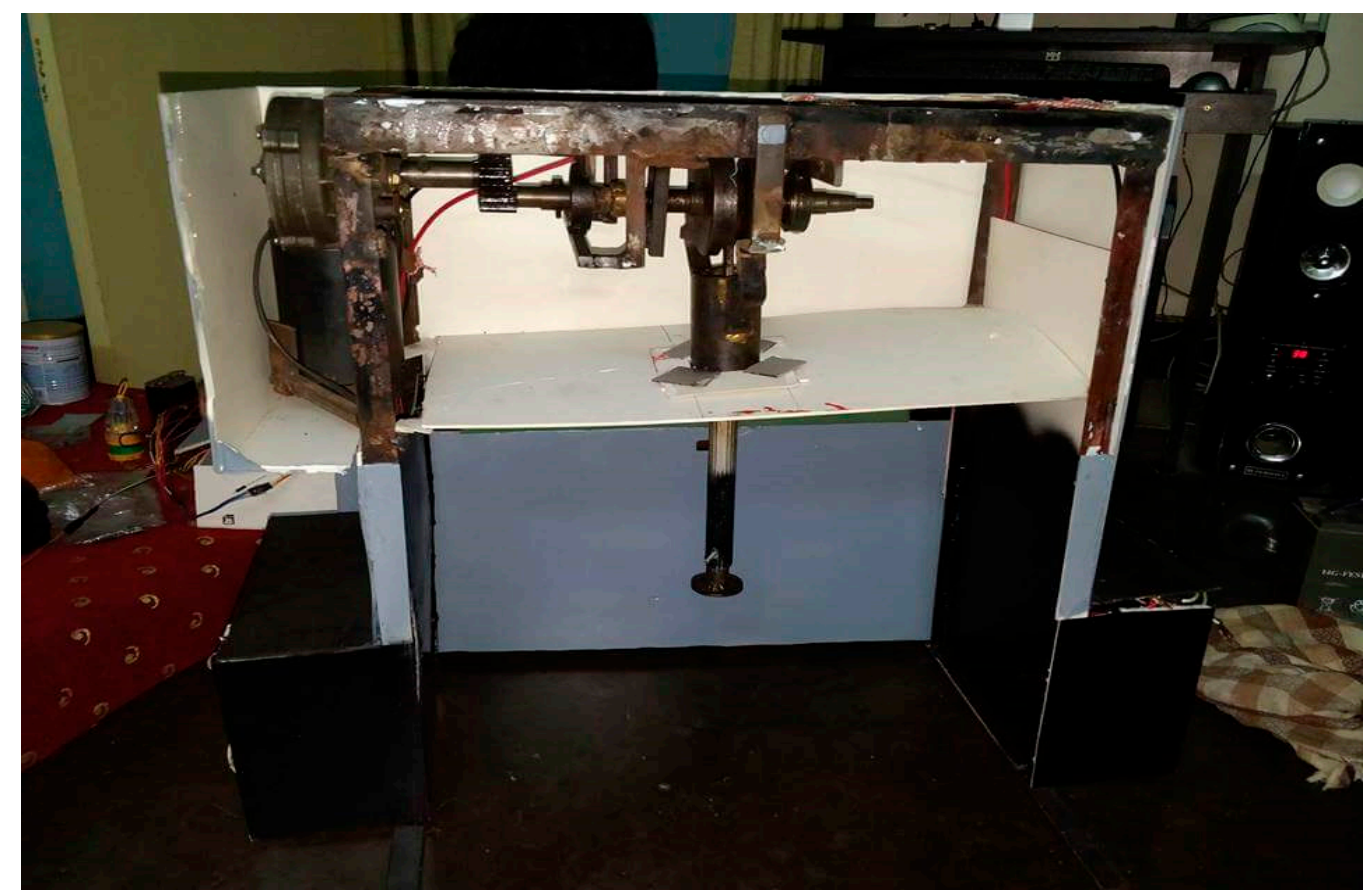

Figure 4. Prototype of the proposed device.

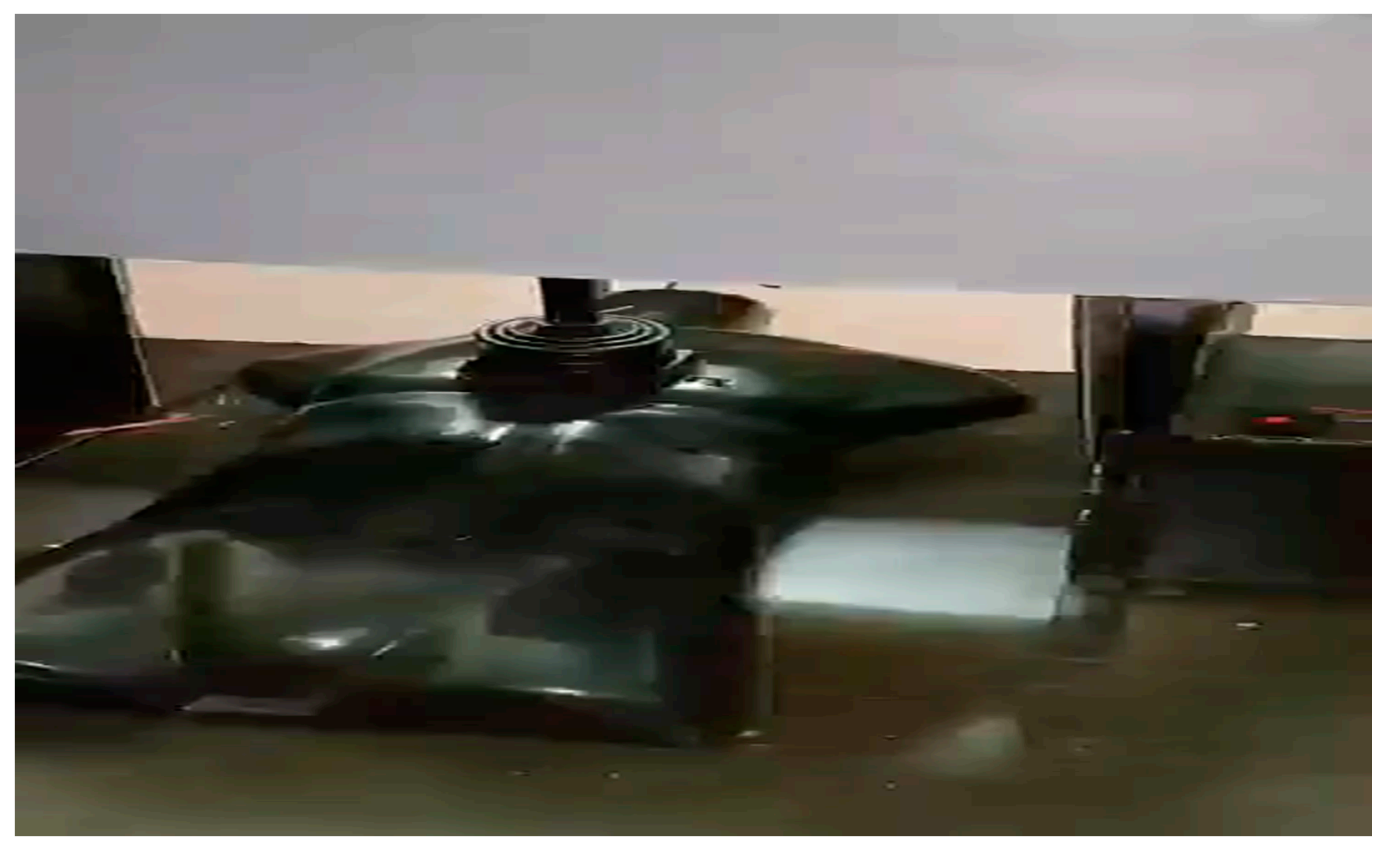

Figure 5. Final prototype of the proposed device testing on dummy. 
After testing it on the human-like dummy, the device was tested on a real human test subject. Figure 5 provides the finished prototype of the recommended machine being tested on the dummy. In Figure 5, the top part of the device appears gray because the internal part of the system was covered with a light gray color board. Figure 6 shows the final prototype of the proposed device being tested on a real human test subject. The device has been tested on 10 different real human volunteers to test the reliability of the performance of the device. An example of testing on one real human test subject is demonstrated in Figure 6. After testing on a human test subject, it was observed that the device worked successfully according to our design requirements. Additional smart features have been added with this device. Due to the limited number of pages, more technical details and further discussion were not possible to provide in this paper. However, the authors of the paper are writing an article with more details of the device which will be submitted to MDPI for review.

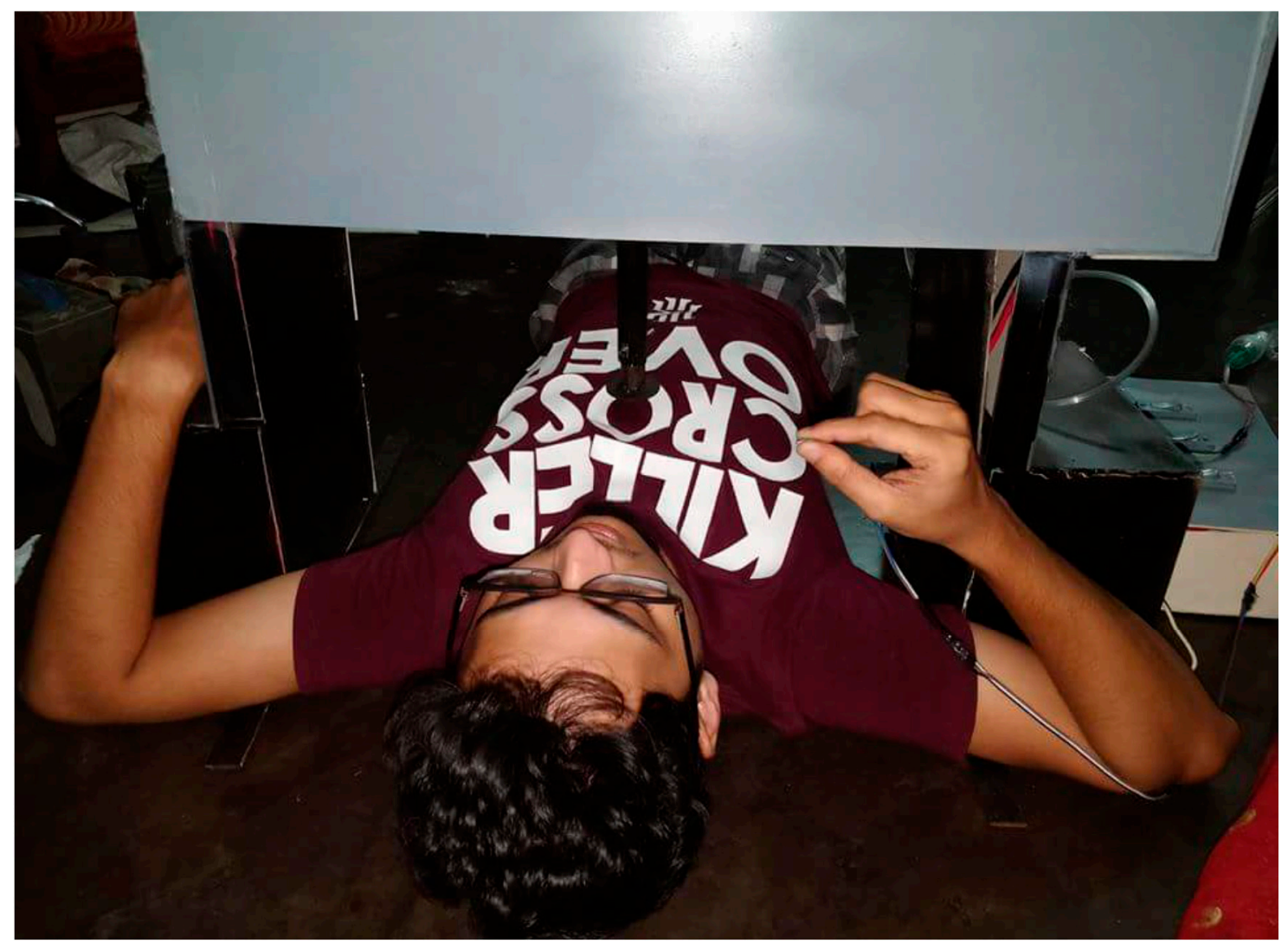

Figure 6. Final prototype of the proposed device testing.

\section{Discussion}

The proposed device will be made to look more user-friendly. An outer and inner side mold will be designed for a real-life appearance. The development cost of the proposed CPR is BDTAKA 42,000 , which is equivalent to USD 500. The same type of devices overseas cost in the range of USD 15,000 to USD 20,000. The proposed device, on the other hand, costs only USD 500, which is much lower in price. This cost-effective device can be easily used by any hospital or emergency services, such as ambulances, and could save a lot of lives. This CPR is automated, portable, and very user friendly. This is a very cost-effective product and people can easily afford to buy it. The proposed solution of the stated problem is innovative because it has never been tried before in Bangladesh. The product will be developed as a common platform for both medical and non-medical people. Currently we are working on the device, to make it more light-weight using different battery systems, such as lithium ion, and adding new features. Next, the device will be tested on a dummy and it will be submitted to BMRC for final approval. This product will be sustainable as we know the users of this device are cardiovascular patients and their numbers are huge. By selling the product, money will be generated, which will make the device more sustainable. Due to the limited number 
of pages, further technical details, results and discussions are not provided in this paper. However, an article summarizing all the results and findings will be written to be submitted in the Applied Science journal with MDPI. The authors of the paper are also working on developing a similar product for children.

\section{Conclusions}

The design prototype, operation principles, testing results and analysis of a low-cost automated CPR device made using locally available raw materials for the treatment of cardiac arrest patients are demonstrated. The automated low-cost CPR device can perform constant and proper chest compressions as required for cardiac arrest patients, which may improve their survival rate. This device is very user friendly, and anyone with basic educational knowledge can operate it. It will increase the life expectancy of patients with cardiovascular disease. The automated CPR device will be very helpful for people in the world. Due to the low cost of the developed automated CPR device, many users can afford to buy it. As mentioned earlier, similar devices abroad cost in the range of USD 15,000 to USD 20,000. However, the proposed device costs only USD 500, which is much lower in price. It can be easily used by any hospital, or emergency services such as ambulances, and could save many lives.

Author Contributions: Both authors have contributed to the development, integration, financing, and testing of the device. This paper has been written by the main author. However, inputs also have been received from co-author in terms of the review and editing of the paper. Both authors of this paper have agreed to publish the paper.

Funding: This research received no external funding.

Acknowledgments: The authors would like to thank Department of Electrical and Computer Engineering of North South University. The research work was carried out in the Engineering Laboratory at the Department of Electrical and Computer Engineering at North South University, Bashundhara, Dhaka, Bangladesh.

Conflicts of Interest: The authors declare no conflict of interest.

\section{References}

1. World Health Organization. Cardiovascular Diseases (CVDs). 2018. Available online: http://www.who.int/mediacentre/factsheets/fs317/en/ (accessed on 15 March 2020).

2. World Health Organization. Noncommunicable Diseases Country Profiles 2014. 2018. Available online: http://www.who.int/nmh/publications/ncd-profiles-2014/en/ (accessed on 15 March 2020).

3. Mozaffarian, D.; Benjamin, E.; Go, A.; Arnett, D.; Blaha, M.; Cushman, M.; Das, S.; de Ferranti, S.; Després, J.; Fullerton, H.; et al. Heart Disease and Stroke Statistics-2016 Update. Circulation 2015, 133, e38-e360.

4. Nassar, B.; Kerber, R. Improving CPR Performance. Chest 2017, 152, 1061-1069.

5. LUCAS ${ }^{\circledR}$-Physio Control. Available online: https://www.physio-control.com/uploadedFiles/Physio85/Contents/Emergency_Medical_Care/Products/ Brochures/LUCAS_BrochuBr_3303294_B.pdf (accessed on 16 May 2020).

6. Zoll.Com. $2018 . \quad$ Available online: https://www.zoll.com/-/media/public-site/products/autopulse/12555-001_rev_5_autopulse_model_100_us er_guide.ashx (accessed on 16 May 2020).

7. Song, Y. T.; Chee, Y. The development of feedback monitoring device for CPR. In Proceedings of the 2011 Annual International Conference of the IEEE Engineering in Medicine and Biology Society, Boston, MA, 30 August-3 September 2011; pp. 3294-3297. doi:10.1109/IEMBS.2011.6090894.

Publisher's Note: MDPI stays neutral with regard to jurisdictional claims in published maps and institutional affiliations. 